\title{
Differentially expressed miRNAs in hepatocellular carcinoma cells under hypoxic conditions are associated with transcription and phosphorylation
}

\author{
BO HU $^{1 *}$, WEI-GUO TANG $^{1 *}$, JIA FAN $^{1,2}$, YANG XU ${ }^{1}$ and HAI-XIANG SUN ${ }^{1}$ \\ ${ }^{1}$ Liver Cancer Institute, Zhongshan Hospital; ${ }^{2}$ Institute of Biomedical Sciences, Fudan University, \\ Key Laboratory of Carcinogenesis and Cancer Invasion, Ministry of Education, Shanghai 200032, P.R. China
}

Received November 18, 2016; Accepted September 13, 2017

DOI: $10.3892 / \mathrm{ol} .2017 .7349$

\begin{abstract}
Hypoxia is a critical aspect of tumor biology and has been associated with poor prognosis and resistance to traditional therapy. In the present study, differentially expressed genes and microRNAs (miRNAs/miRs) were screened for in the hepatocellular carcinoma (HCC) cell line Huh7 under hypoxic conditions. On the basis of microarray data, 11,508 mRNAs and 58 miRNAs exhibiting $\geq 1.5$-fold change in expression under hypoxic conditions were identified. Gene Ontology (GO) and Kyoto Encyclopedia or Genes and Genomes pathway analysis revealed that the differentially expressed genes were primarily involved in cell cycle regulation, cell division, transcription and G-protein-coupled receptor signaling pathways. Using the TargetScan and miRanda software packages with the miRNA-mRNA negative expression network, differentially expressed miRNA targets were predicted. GO analysis revealed that the primary function of these miRNAs was to regulate transcription and phosphorylation. The miRNA-mRNA networks for transcription and phosphorylation were analyzed. Network analysis revealed that the key miRNAs in these networks were miR-19a, miR-34a, miR-29a, mir-196a, miR-25 and miR-1207, whose potential gene targets include DNA-binding proteins, zinc-finger proteins and transcription factors. Certain protein
\end{abstract}

Correspondence to: Dr Hai-Xiang Sun or Dr Yang Xu, Liver Cancer Institute, Zhongshan Hospital, Fudan University, Key Laboratory of Carcinogenesis and Cancer Invasion, Ministry of Education, 136 Yi Xue Yuan Road, Shanghai 200032, P.R. China

E-mail: sun.haixiang@zs-hospital.sh.cn

E-mail: drxuyang@gmail.com

${ }^{*}$ Contributed equally

Abbreviations: miRNA, microRNA; HCC, hepatocellular carcinoma; GO, Gene Ontology; KEGG, Kyoto Encyclopedia of Genes and Genomes; HIFs, hypoxia-inducible factors; RT-qPCR, reverse transcription-quantitative polymerase chain reaction

Key words: hepatocellular carcinoma, hypoxia, microRNA, transcription, phosphorylation kinases, includingmitogen-activated protein kinase (MAPK) 1, MAPK kinase kinase4 and cyclin-dependent kinase 18, were also revealed to be present in the network. In hypoxic HCC tissue, levels of several key miRNAs implicated in the network analyses (miR-19a, miR-34a, miR-25 and miR-1207) were revealed to exhibit increased expression levels compared with the surrounding tissue. The results of the present study provide evidence that miRNAs serve an important function in transcription and phosphorylation in the hypoxic response of HCC cells.

\section{Introduction}

The hypoxic microenvironment serves a function in tumor growth, metastasis and recurrence, as tumors with extensive low oxygen tension tend to lead to a poor prognosis and exhibit resistance to conventional therapies (1). The underlying molecular mechanisms of the cellular response to oxygen deprivation have been the subject of numerous studies and are known to be complex. Hypoxia-inducible factors (HIFs) regulate $>100$ genes in response to a decrease in oxygen and are known to serve a function in the hypoxic condition (2). MicroRNAs (miRNAs) have also been demonstrated to be involved in the process of cellular adaptation to the hypoxic microenvironment (3). miRNAs are non-coding single-stranded RNAs of $\sim 22$ nucleotides that mediate sequence-dependent post-transcriptional negative regulation of gene expression, and are known to serve a function in fundamental processes including cell proliferation (4), metabolism (5) and cancer metastasis (6), in addition to canonical signaling pathways including Notch, mitogen-activated protein kinase (MAPK) (7) and the Toll-like receptor signaling pathways $(7,8)$. The study of the biological significance and utility of miRNAs is an expanding field, and it is increasingly evident that miRNAs serve complex functions and diverse functions specific to individual miRNA sequence, cell type and tissue environment. Although there are reports of hypoxia-regulated miRNAs in several types of tumor, to the best of our knowledge, cell-specific regulation and disease-specific alterations of miRNA in hepatocellular carcinoma (HCC) have yet to be investigated. In the present study, mRNA and miRNA expression profile changes were analyzed using microarray technology to uncover the 
underlying function of miRNA in the adaptation of HCC cells to the hypoxic microenvironment.

\section{Materials and methods}

Cell culture. Huh7 cells, purchased from the Institute of Biochemistry and Cell Biology (Shanghai, China), were maintained in Dulbecco's modified Eagle's medium (Thermo Fisher Scientific, Inc., Waltham, MA, USA) supplemented with $10 \%$ fetal bovine serum. Cells were incubated at $37^{\circ} \mathrm{C}$ with $5 \% \mathrm{CO}_{2}$. To create hypoxic conditions, cells $12 \mathrm{~h}$ post-seeding were placed in a hypoxia workstation (In vivo 200, Ruskinn Technology, Ltd., Bridgend, UK) for $48 \mathrm{~h}$ at $1 \% \mathrm{O}_{2}, 5 \% \mathrm{CO}_{2}$ and $37^{\circ} \mathrm{C}$.

Extraction and labeling of sample RNA. Total RNA was extracted and purified using TRIzol ${ }^{\circledR}$ reagent (Thermo Fisher Scientific, Inc.), according to the manufacturer's protocol. RNA integration was verified using an Agilent Bioanalyzer 2100 (Agilent Technologies, Inc., Santa Clara, CA, USA). miRNA in the total RNA was labeled using the miRNA Complete Labeling and Hybkit (Agilent Technologies, Inc.), according to the manufacturer's protocol.

Array hybridization, image scanning and bioinformatic data processing. Each slide was hybridized with 100 ng cyanine 3-labeled RNA using the miRNA Complete Labeling and Hybkit in a hybridization oven (Agilent Technologies, Inc.) at $55^{\circ} \mathrm{C}, 20$ revolutions/min for $20 \mathrm{~h}$, according to the manufacturer's protocol. Following hybridization, slides were washed in staining dishes (Thermo Fisher Scientific, Inc.) with Gene Expression Wash Buffer kit (Agilent Technologies, Inc.). Slides were scanned using an Agilent Microarray Scanner (Agilent Technologies, Inc.) and read using Feature Extraction Software (version 10.7; Agilent Technologies, Inc.) using default settings. Raw data were normalized using the Quantile algorithm with GeneSpring software (version 12.6; Agilent Technologies, Inc.).

Gene Ontology (GO) and Kyoto Encyclopedia of Genes and Genomes (KEGG) analysis. Using the GO and KEGG databases, functional classifications and pathways of differentially expressed genes were analyzed. TargetScan and miRanda databases were used to analyze differentially expressed miRNAs and their associated negatively regulated genes, and the GO and KEGG pathway labels were applied to determine the significant functions of the differentially expressed miRNAs $(9,10)$. Fisher's exact test was applied to identify the significant GO categories and the false discovery rate was used to correct the P-values.

miRNA-target network. The association between the miRNAs and their gene targets was analyzed on the basis of differential expression values, and an miRNA-target network was built on the interactions of miRNA and genes identified in the Sanger miRNA database (miRBase.org). Circles represent genes and arrows represent miRNAs, and their association is presented as an edge. The size of the arrow changes on the basis of the degree of contribution of the miRNA to gene expression, where key miRNAs in the network are presented as larger arrows.
Reverse transcription-quantitative polymerase chain reaction $(R T-q P C R)$. Certain genes and miRNAs identified in these analyses were selected for verification using RT-qPCR.

cDNA was synthesized using random primers and PCR was performed using a SYBR master mix (Thermo Fisher Scientific, Inc.), according to the manufacturer's protocol; GAPDH was used as a control. The primer sequences were used as follows: RAP2A; forward: 5'-GACCCCACCATC GAGGACTTCTAC-3', reverse, 5'-TGGCACTTTCTCATA CCGCTTCAC-3';. GPR4; forward, 5'-TCCCTAACTGCG CTGTGTCCTATC-3'; reverse, 5'-ATCGCTGGGCAATGG TGAGAA-3'; RASA4; forward, 5'-GGCAGGGCTTTTGTG GGTTATG-3', reverse, 5'-GCTGGGAGGGAGGAGGCTTTA G-3'; RAPGEF2; forward, 5'-CAGTGGATTCCGAAGACG ACGAC-3', reverse, 5'-CCACCACTGCGAACACCATCAC-3'; METTL20; forward, 5'-AGAGGCTTAATCATTGGGCAC TG-3', reverse, 5'-CGAAAAGCATACAACACGCAAGAA-3'; RAPH1; forward, 5'-TCCCCCTACCCCTCCTGTTCC-3', reverse, 5'-ACTGGCTGGCTATCTGCTTCACG-3'; CXCL-2; forward, 5'-TGCGCCCAAACCGAAGTCATA-3', reverse, 5'-GTGGCCTCTGCAGCTGTGTCTCT-3'; FUBP-1; forward, 5'-ACACCCGAAAGGATAGCAC-3', reverse, 5'-TTGCCT TGACCTCTACCTC-3'; YAP; forward, 5'-AGGAGAGGC TGCGGCTGAAAC-3', reverse, 5'-TGAGACATCCCGGGA GAAGACACT-3'; GAPDH; forward, 5'-GGGGCTCTCCAG AACATCATCC-3', reverse, 5'-ACGCCTGCTTCACCACCT TCTT-3'. The miRNA-19a (assay ID 002424; Thermo Fisher Scientific, Inc.), miR-34a (assay ID 000425; Thermo Fisher Scientific, Inc.), miR-25 (assay ID 000403; Thermo Fisher Scientific, Inc.) and miR-1207 (assay ID 241060; Thermo Fisher Scientific, Inc.) expression levels were measured using TaqMan $^{\mathrm{TM}}$ MicroRNA Assays (cat. no. 4427975; Thermo Fisher Scientific, Inc.). Thermal cycling parameters were as follows: Polymerase activation at $95^{\circ} \mathrm{C}$ for $10 \mathrm{~min}$, followed by 40 cycles of $95^{\circ} \mathrm{C}$ for $15 \mathrm{sec}$ and $60^{\circ} \mathrm{C}$ for $1 \mathrm{~min}$. The expression levels of miRNAs were analyzed using the TaqMan miRNA assays and TaqMan miRNA RT kit (Applied Biosystems; Thermo Fisher Scientific, Inc.) on an Applied Biosystems 7500 RT-qPCR system (11).

\section{Results}

Differential gene expression under normoxia and hypoxia. To investigate gene expression profile changes in hypoxic cells, microarray analysis was conducted on Huh7 cells after $48 \mathrm{~h}$ of culture under hypoxic conditions. Among the transcripts tested, the expression levels of 4,146 genes were identified to be upregulated and those of 7,362 genes were identified to be downregulated (fold change $>1.5$; $\mathrm{P}<0.05)$. These differentially expressed genes included HIFs, platelet-derived growth factor subunit B, Krüppel-like factor 8, C-X-C motif chemokine ligand 5 and ribonucleotide reductase catalytic subunit $\mathrm{M}$, which are known to be involved in cell cycle regulation, transcription and apoptosis (Fig. 1A). GO analysis was performed on upregulated and downregulated genes. Downregulated genes were primarily identified to be involved in the cell cycle, cell division and transcription, whereas upregulated genes were typically associated with G-protein-coupled receptor signaling pathways, signal transduction, response to stimuli and synaptic 

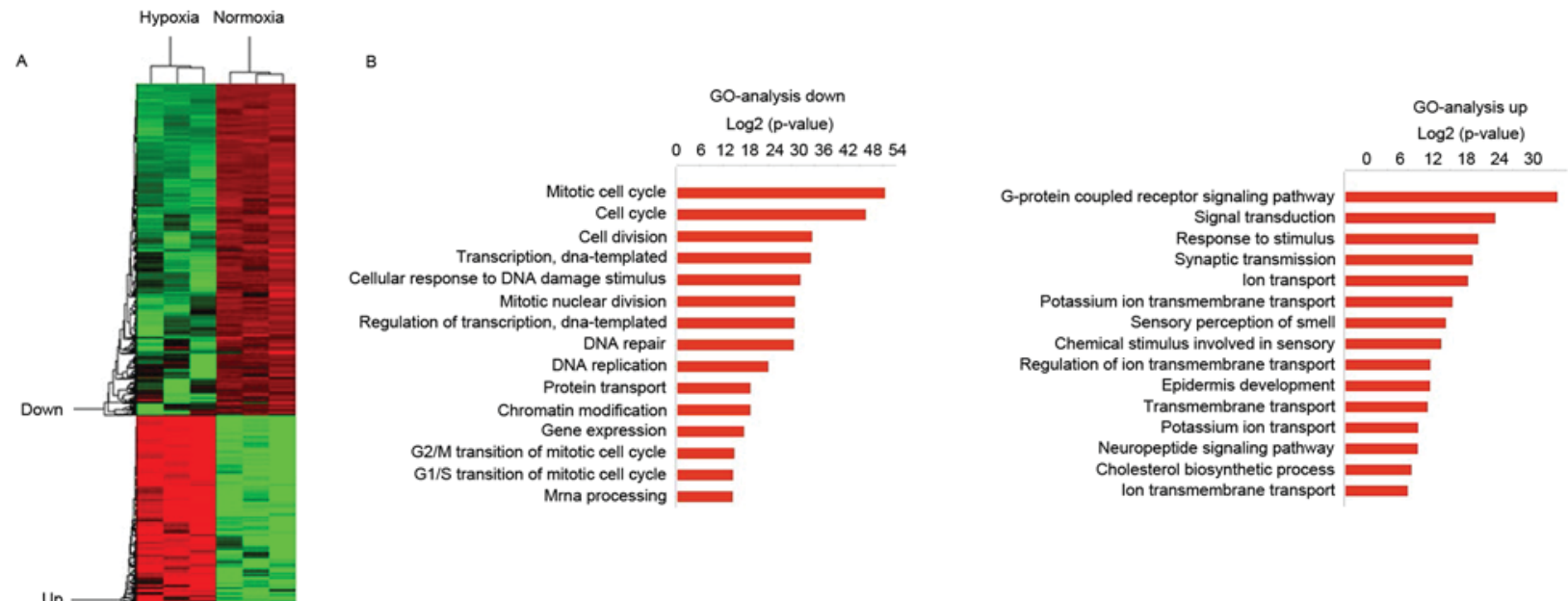

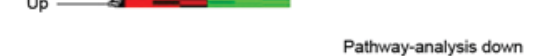

c

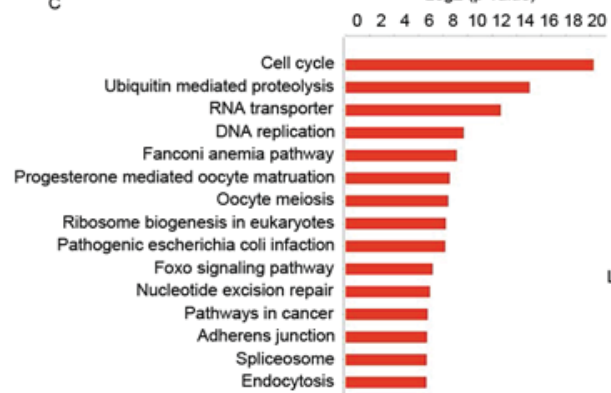

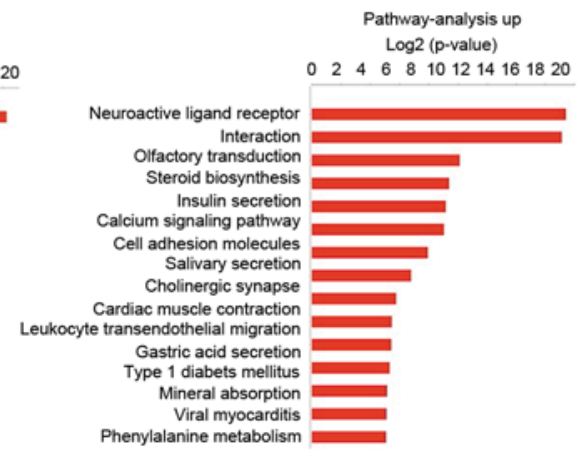

D

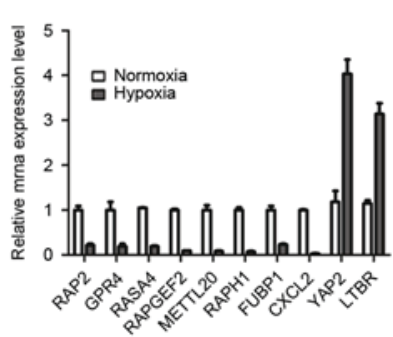

Figure 1. Differentially expressed mRNAs in Huh7 cells after $48 \mathrm{~h}$ under hypoxia. (A) A heat map presents differential gene expression patterns (fold change $>2$ or $<0.5$ ). (B) GO analysis of downregulated and upregulated mRNAs, respectively. (C) Pathways of downregulated and upregulated mRNAs, respectively. (D) Reverse transcription-quantitative polymerase chain reaction verification of the selected genes under normal and hypoxic conditions. GO, Gene Ontology; RAP2, Ras-related protein Rap-2a; GPR4, G-protein-coupled receptor 4; RASA4, Ras GTPase-activating protein 4; RAPGEF2, Rap guanine-nucleotide-exchange factor 2; METTL20, methyltransferase-like 20; RAPH1, Ras-associated and pleckstrin homology domain-containing protein 1; FUBP1, far-upstream element-binding protein 1; CXCL2, C-X-C motif chemokine ligand 2; YAP2, yes-associated protein 1; LTBR, lymphotoxin $\beta$ receptor.

transmission (Fig. 1B). KEGG pathway analysis revealed that downregulated genes were primarily associated with cell cycle, ubiquitin-mediated proteolysis, RNA transport and DNA replication, and the upregulated genes were associated with neuroactive ligand-receptor interaction, steroid biosynthesis, insulin secretion and the calcium signaling pathway (Fig. 1C). Together, the GO and KEGG pathway analysis results revealed that the annotated differentially expressed genes were involved in a wide variety of signaling pathways.

Huh7 cells were identified to exhibit a number of changes, including changes in the cell cycle, DNA replication and transcription in response to the hypoxic conditions. RT-qPCR was performed in order to confirm the gene expression changes observed in the microarray analysis. The results revealed that Ras-related protein Rap-2a, G-protein-coupled receptor 4, Rasp21 protein activator 4, Rap guanine-nucleotide-exchange factor 2, methyltransferase-like 20, Ras-associated and pleckstrin homology domain-containing protein 1, far-upstream element-binding protein 1 and $\mathrm{C}-\mathrm{X}-\mathrm{C}$ motif chemokine ligand 2 expression were significantly decreased, and the expression of yes-associated protein 1 and lymphotoxin $\beta$ receptor was significantly increased. These results demonstrated a trend that was consistent with the results of microarray analysis (Fig. 1D). hypoxic and normoxic conditions. Microarray analysis demonstrated that 58 miRNAs were differentially expressed under hypoxic conditions. Among these, 35 miRNAs were revealed to be downregulated and 23 were identified to be upregulated (fold change $>1.5 ; \mathrm{P}<0.05$; Fig. $2 \mathrm{~A}$ ). The 10 most upregulated miRNAs were miR-574-3P, miR-320d, miR-151a-5p, miR-374b-5p, miR-19a-3p, miR-1246, miR-122-3p, miR-122-5p, miR-3132 and miR-29b-3p, and the 10 most downregulated miRNAs were miR-3926, miR-3138, miR-150-3p, miR-4252, miR-762, miR-6090, miR-3934-5p, miR-3960, miR-4430 and miR-4443; all these miRNAs exhibited a fold change greater than 3 in hypoxic cells. Differentially expressed miRNAs were confirmed using RT-qPCR. Among the validated miRNAs, 3 were demonstrated to be significantly different between hypoxic and normoxic conditions, of which one exhibited a similar expression change to that observed using microarray analysis, although no significant difference was observed (Fig. 2B).

Using the TargetScan and miRanda software packages, differentially expressed miRNA targets were predicted. Predicted genes, which exhibit negative co-expression with differentially expressed miRNAs, were used to analyze the miRNA gene regulation network. GO analysis revealed that the primary functions of the differentially expressed miRNAs were as positive and negative regulators of transcription from the RNA polymerase promoter, phosphorylation and regulation of transcription. These results demonstrate that the majority of 

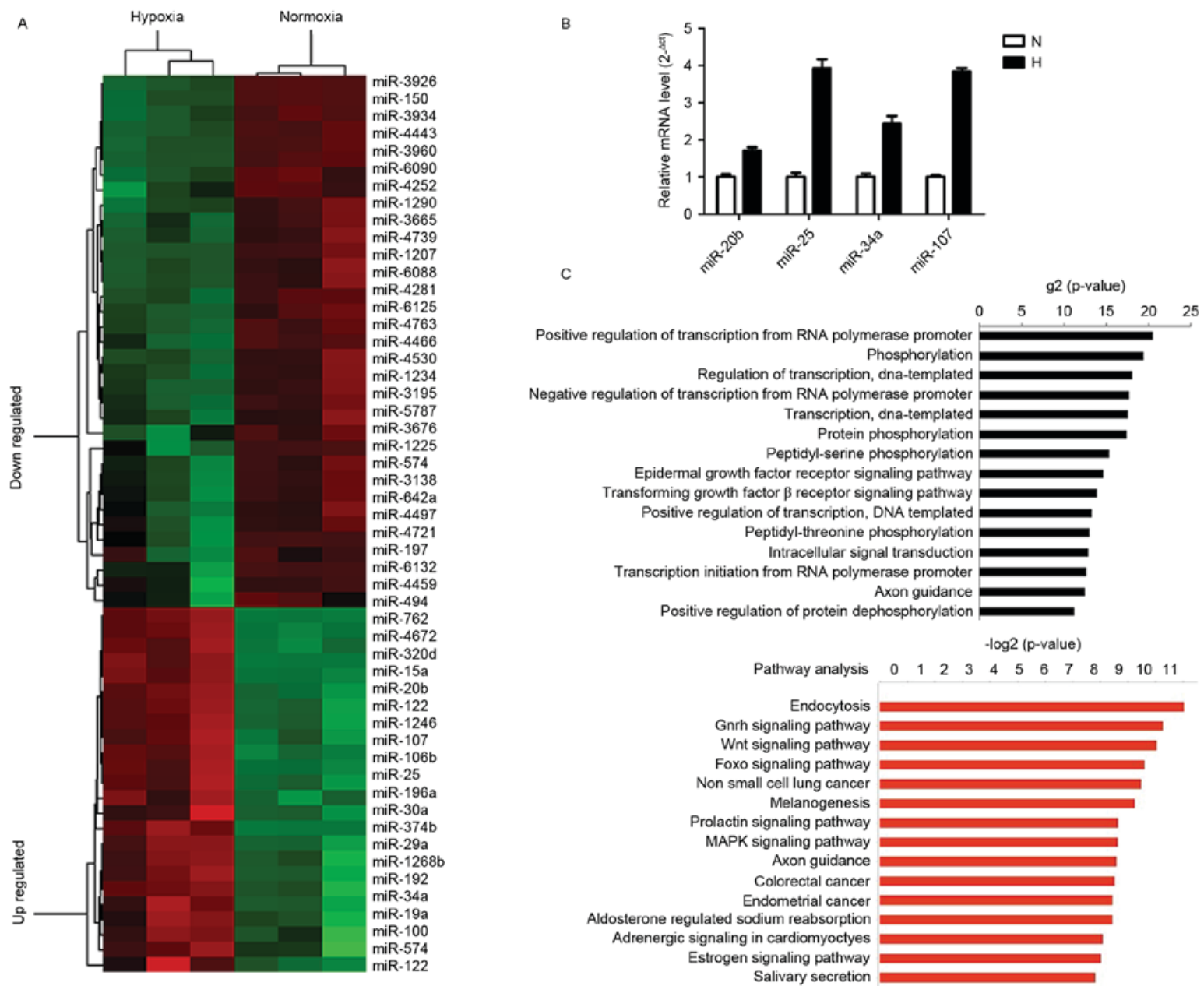

Figure 2. Differentially expressed miRNAs in Huh7 cells after 48 hunder hypoxia and their GO and KEGG pathway analysis. (A) A heat map presents the differentially expressed miRNA (fold change $>2$ or fold change $<0.5$ ). (B) Selected differentially expressed miRNAs were confirmed using the reverse transcription-quantitative polymerase chain reaction. (C) GO and KEGG pathway analysis of differentially expressed miRNA target genes. miRNA/miR, microRNA; GO, Gene Ontology; KEGG, Kyoto Encyclopedia of Genes and Genomes; N, normal; H, hypoxia.

differentially expressed miRNAs regulate transcription-modifying and phosphorylation-associated genes. KEGG pathway analysis revealed that genes associated with endocytotic, gonadotropin-releasing hormone, wingless-related integration site and forkhead box O (FOXO) signaling pathways were involved in the 15 most altered pathways list (Fig. 2C). These associated signaling pathways are known to exhibit aberrant activation in HCC and serve crucial functions in the regulation of cellular proliferation, apoptosis, stem and progenitor cell expansion, and drug resistance.

Significance of miRNA function on transcription and phosphorylation. From the aforementioned results, it was identified that the majority of differentially expressed miRNAs were involved in the regulation of transcription and modification of phosphorylation. Transcription is the initial step of gene expression, and is performed in the nucleus by RNA polymerase, which requires a promoter sequence and a set of DNA-binding proteins to initiate the process. Potential target genes of the differentially expressed miRNAs were revealed to be positive and negative regulators of transcription from the RNA polymerase promoter. It was identified that certain DNA-binding proteins including transcriptional regulating factor 1,inhibitor of DNA binding 2 and zinc-finger proteins 1-3, all of which are required for gene transcription, had a distinct expression profile under hypoxic conditions, and may be the potential targets of several of the identified miRNAs (miR-19a, miR-196a, miR-192 and miR-34a). Certain transcription factors, including mothers against decapentaplegic homolog 4 , E2 factor (E2F), specificity protein 1 and Krüppel-like factor 13, also exhibited marked expression fold changes under hypoxic conditions and were revealed to control the rate of transcription. Differentially expressed miRNAs identified under hypoxic conditions (including miR-19a, miR-34a and miR-122) are known to be involved in p53 $(12,13)$, c-myc (14) and E2F (15) signaling pathways. These miRNAs associated with cell proliferation and epithelial-mesenchymal transition $(16,17)$ were identified in the miRNA-mRNA network and had potential targets genes including RNA polymerase elongation factor II, RNA-binding motif, single-stranded-interacting protein 1, and signal transducer and activator of transcription 3, all of which regulate gene transcription (Fig. 3). The miRNA-mRNA negative expression network also revealed that a large portion of mRNA was downregulated, whereas the majority of the associated miRNA was upregulated, compared with control (normoxic) conditions (Fig. 3). 


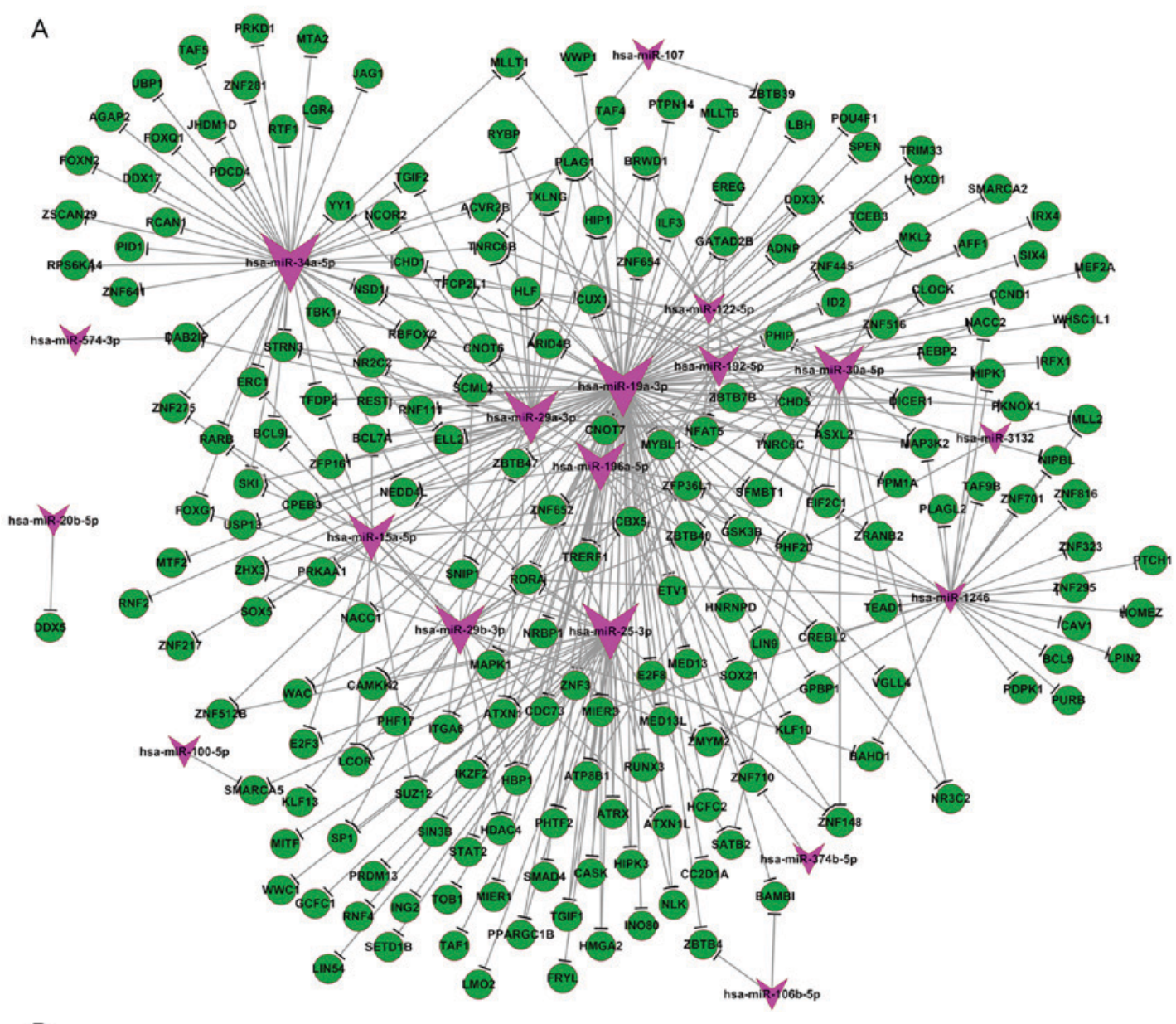

B

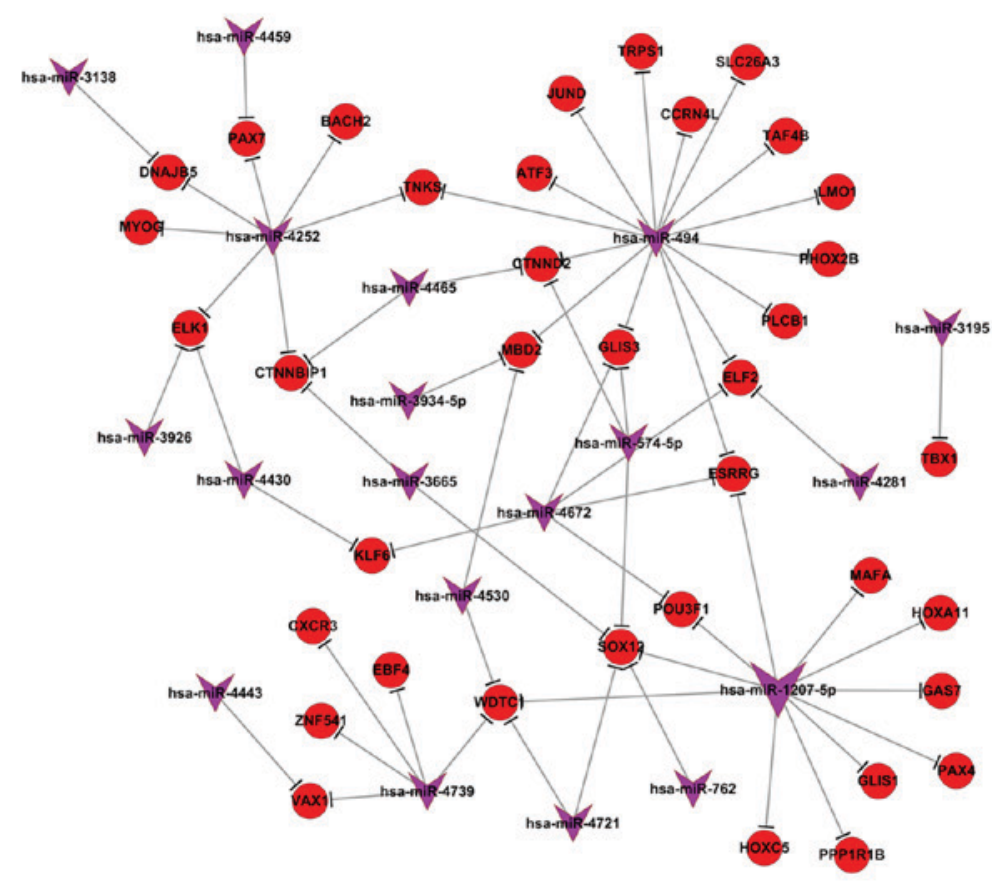

Figure 3. miRNA-mRNA negative network of differentially expressed miRNAs associated with the transcription pathway. (A) miRNAs with increased expressed and their target genes. (B) Downregulated miRNA and the associated transcription pathway. miRNA/miR, microRNA.

Phosphorylation is a post-translational protein modification, which activates enzymes, alters their function and activity and serves a function in a wide range of cellular processes. In hypoxia, the majority of genes associated with phosphorylation [including MAPK1, MAPK kinase kinase (MAP3K)4 and phosphoinositide 3-kinase C2 domain-containing $\alpha$ polypeptide] were downregulated, and a number of genes [including cyclin-dependent kinase (CDK) 18, proto-oncogene 


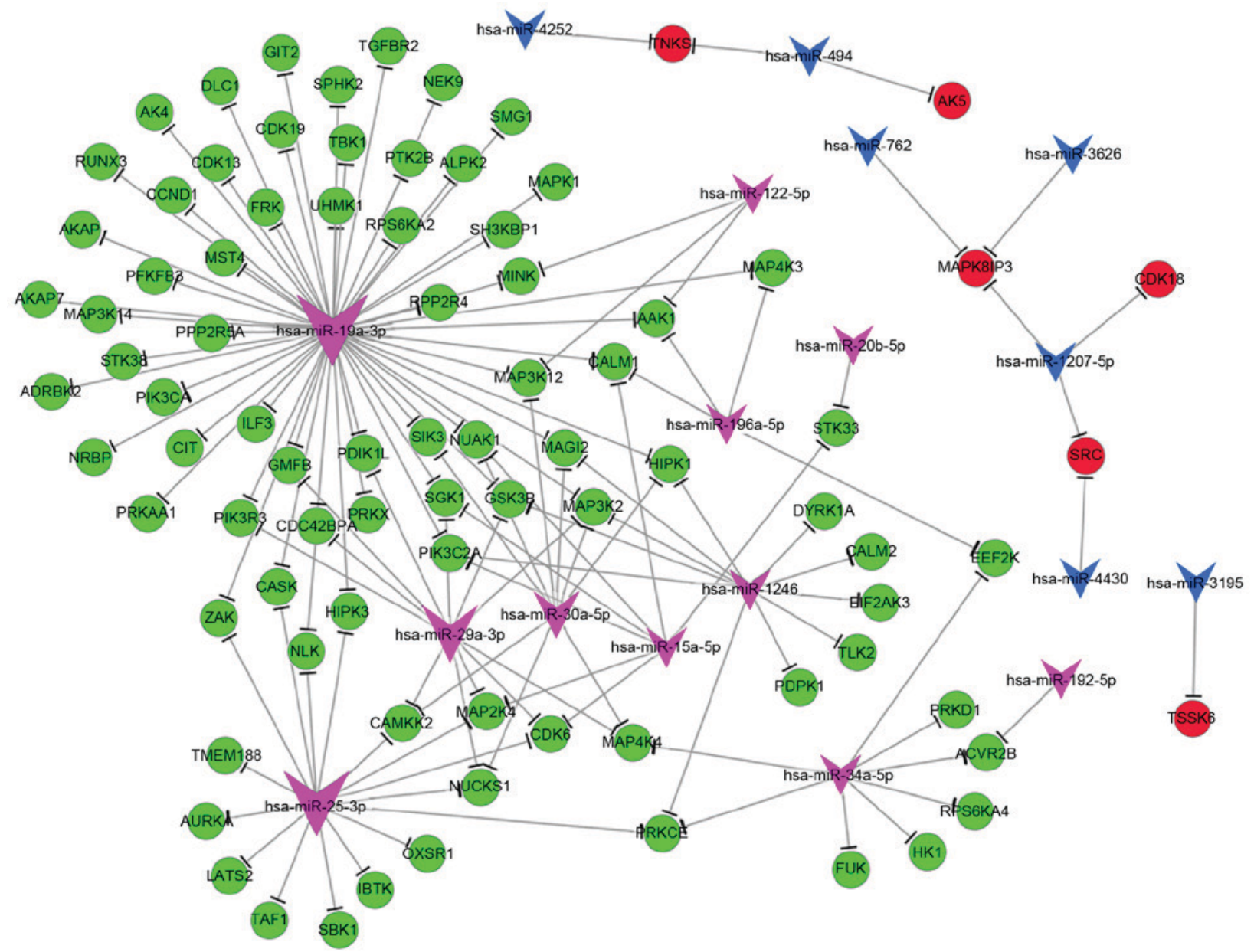

Figure 4. miRNA-mRNA negative network of differentially expressed miRNAs associated with phosphorylation modification. miRNA/miR, microRNA.

miR-34a

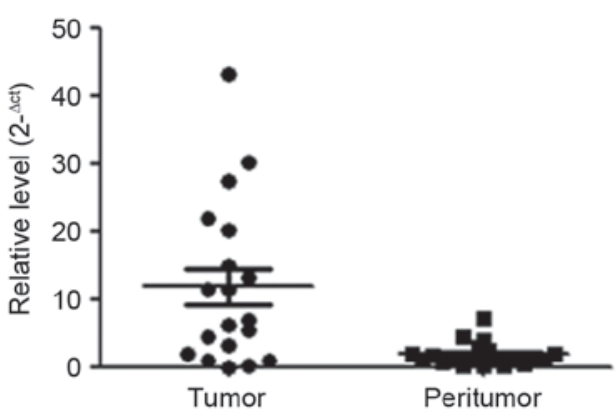

miR-25

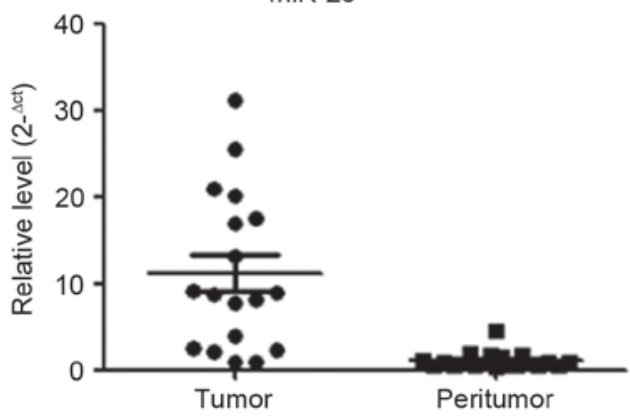

miR19a

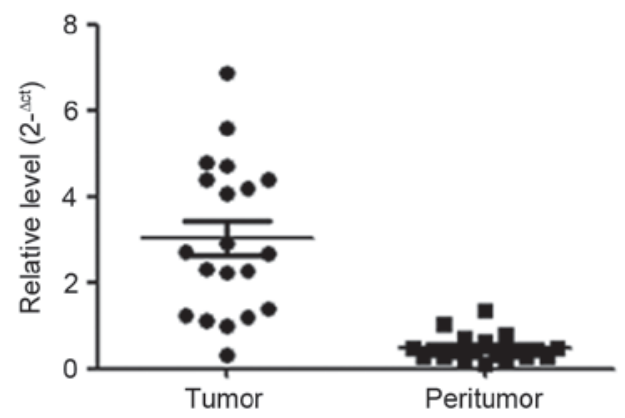

miR-1207

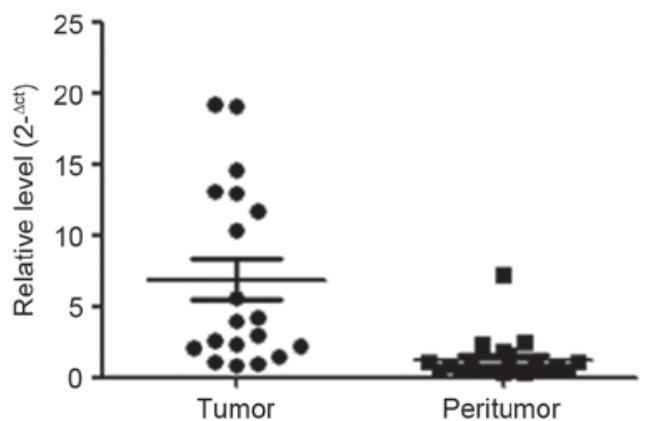

Figure 5. Expression levels of selected miRNAs in hepatocellular carcinoma tumor and peritumor tissue. miRNA/miR, microRNA. 
tyrosine-protein kinase and MAPK8-interacting protein 3] were upregulated. The GO analysis revealed that differentially expressed miRNAs serve a function in phosphorylation. Upon analyzing the miRNA-mRNA network of phosphorylation, the key miRNAs were identified to be miR-19a, miR-25, miR-29 and miR-34a, and their potential target genes were MAP3K12, MAPK1, CDK1 and phosphoinositide3-kinase regulatory subunit 3 (Fig. 4).

Key miRNA expression in HCC. The aforementioned results identified several miRNAs have a function under hypoxic conditions, whose potential target genes involve phosphorylation and transcription. It was also revealed that key miRNAs miR-34a, miR-19a, miR-25 and miR-1207 exhibited increased expression levels in HCC tissue compared with corresponding peritumor tissue (Fig. 5). As tumor growth and progression results in increasing hypoxia in the tumor microenvironment and the results of the present study demonstrate that the miRNAs which increased under hypoxic conditions are increased in tumor tissue, it is possible that these identified miRNAs are of clinical importance.

\section{Discussion}

miRNAs are regulators of tumorigenesis that regulate gene expression on the basis of sequence complementarity, particularly between the seed region and the $3^{\prime}$ untranslated regions of the target. Dysregulation of miRNA expression has been observed in almost all neoplasms, demonstrating the importance of miRNAs in tumor biology. Notably, different types of cancer tend to exhibit specific expression patterns and the underlying molecular mechanisms of these shifts in the tumor profile remain unresolved $(18,19)$. A previous study has demonstrated that miRNA levels differ in low oxygen and contribute to the regulation of specific genes under hypoxic conditions (20). In the present study, the altered expression of miRNAs under hypoxic pressure was examined in an HCC cell line and it was revealed that 58 miRNAs were aberrantly expressed in hypoxia, a number of which (including miR-106, miR-15a and miR-29a) were identified to be associated with cell cycle changes, drug resistance and abnormal expression in certain types of cancer. Additionally, certain key miRNAs were identified in the miRNA-mRNA network of HCC cells in response to hypoxic pressure, including miR-29a, miR-34a, miR-19a and miR-25.

A feature in numerous types of cancer, including HCC, hypoxia results in the expression of specific miRNAs, referred to as hypoxamirs (21). A large number of hypoxamirs have been identified in retinoblastoma, glioblastoma $(21,22)$ and other types of tumor. In the present study, hypoxamirs from an HCC cell line were identified. Normal expression of certain miRNAs in liver cells was revealed to be altered. For example, miR-122 is the most highly abundant miRNA expressed in hepatocytes, where it has been demonstrated to regulate hepatic cholesterol and lipid metabolism, and aberrant expression leads to the development of fibrosis and $\operatorname{HCC}(23,24)$. Under hypoxic conditions miR-122-3p and miR-122-5p were upregulated by a factor of $>2$. Additionally, the expression of certain key miRNAs was tested in HCC tumor tissue compared with their corresponding peritumor tissue and it was revealed that expression levels were consistently changed as a result of the hypoxic conditions in the tumor. Hypoxamirs in the HCC cell may be used as a library to screen miRNAs as potential biomarkers and therapeutic targets.

Since each miRNA potentially regulates a large number of targets, understanding the function of miRNAs in tumorigenesis may provide insight into the key questions that remain in cancer research. In the present study, mRNA and miRNA expression changes induced by hypoxic conditions in Huh7 HCC cells were evaluated. It was observed that mRNA and miRNA undergo changes in expression profiles, and GO analysis revealed that the majority of these changes are involved in transcriptional regulation and phosphorylation, particularly the differentially expressed miRNA. In total, $40 \%$ of the 15 most differentially expressed miRNAs $(6 / 15)$ are involved in regulating transcription. AnmiRNA-mRNA negative association network analysis revealed that differentially expressed microRNA target genes include zinc-finger proteins (ZNF445, ZNF64, etc.), transcription factors (neural precursor cell expressed developmentally downregulated protein 4, runt-related transcription factor 3, FOXO1, transcription factor Dp-2, etc.) and others that are known to regulate the rate of transcription. Key miRNAs identified in the present study include miR-34a, miR-19a and miR-25, which exhibited a significantly increased expression level in HCC tumors compared with their surrounding tissue. Taken together, the results of the present study demonstrate that miRNA serves an important function in tumor biology by regulating a number of transcription factors and targeting transcriptional regulators in HCC cells under hypoxic conditions.

\section{Acknowledgements}

The present study was accomplished through combined grant support from the National Key Sci-Tech Project (grant nos. 2013ZX10002011-004 and 2012ZX0930100-007), National Natural Science Foundation of China (grant nos. 81302100, 81572884 and 81372317), Zhongshan Hospital Outstanding Youth Fund (grant no. 2015ZSYXQN03), the Specialized Research Fund for the Doctoral Program of Higher Education (grant no. 20120071120068) and The Pujiang Scholars Fund of Shanghai (grant no. 13PJD007).

\section{References}

1. Zhou TY, Zhuang LH, Hu Y, Zhou YL, Lin WK, Wang DD, Wan ZQ, Chang LL, Chen Y, Ying MD, et al: Inactivation of hypoxia-induced YAP by statins overcomes hypoxic resistance tosorafenib in hepatocellular carcinoma cells. Sci Rep 6: 30483, 2016.

2. Kai AK, Chan LK, Lo RC, Lee JM, Wong CC, Wong JC and Ng IO: Down-regulation of TIMP2 by HIF-1 $\alpha / \mathrm{miR}-210 / \mathrm{HIF}-3 \alpha$ regulatory feedback circuit enhances cancer metastasis in hepatocellular carcinoma. Hepatology 64: 473-487, 2016.

3. Jia YY, Zhao JY, Li BL, Gao K, Song Y, Liu MY, Yang XJ, Xue Y, Wen AD and Shi L: miR-592/WSB1/HIF-1 $\alpha$ axis inhibits glycolytic metabolism to decrease hepatocellular carcinoma growth. Oncotarget 7: 35257-35269, 2016.

4. Candini O, Spano C, Murgia A, Grisendi G, Veronesi E, Piccinno MS, Ferracin M, Negrini M, Giacobbi F, Bambi F, et al: Mesenchymal progenitors aging highlights a miR-196 switch targeting HOXB7 as master regulator of proliferation and osteogenesis. Stem Cells 33: 939-950, 2015. 
5. Pogribny IP and Beland FA: Role of microRNAs in the regulation of drug metabolism and disposition genes in diabetes and liver disease. Expert Opin Drug Metab Toxicol 9: 713-724, 2013.

6. Krzeszinski JY, Wei W, Huynh H, Jin Z, Wang X, Chang TC, Xie XJ, He L, Mangala LS, Lopez-Berestein G, et al: miR-34a blocks osteoporosis and bone metastasis by inhibiting osteoclastogenesis and Tgif2. Nature 512: 431-435, 2014.

7. Xu G, Zhang Y, Wei J, Jia W, Ge Z, Zhang Z and Liu X: MicroRNA-21 promotes hepatocellular carcinoma HepG2 cell proliferation through repression of mitogen-activated protein kinase-kinase 3. BMC Cancer 13: 469, 2013.

8. Li Y, Shi Y, McCaw L, Li YJ, Zhu F, Gorczynski R, Duncan GS Yang B, Ben-David Y and Spaner DE: Microenvironmental interleukin-6 suppresses toll-like receptor signaling in human leukemia cells through miR-17/19A. Blood 126: 766-778, 2015.

9. Ashburner M, Ball CA, Blake JA, Botstein D, Butler H, Cherry JM, Davis AP, Dolinski K, Dwight SS, Eppig JT, et al: Gene ontology: Tool for the unification of biology. Gene ontology consortium. Nat Genet 25: 25-29, 2000.

10. Draghici S, Khatri P, Tarca AL, Amin K, Done A, Voichita C, Georgescu C and Romero R: A systems biology approach for pathway level analysis. Genome Res 17: 1537-1545, 2007.

11. Livak KJ and Schmittgen TD: Analysis of relative gene expression data using real-time quantitative PCR and the 2(-Delta Delta C(T)) method. Methods 25: 402-408, 2001.

12. Hamilton MP, Rajapakshe K, Hartig SM, Reva B, McLellan MD, Kandoth C, Ding L, Zack TI, Gunaratne PH, Wheeler DA, et al: Identification of a pan-cancer oncogenic microRNA superfamily anchored by a central core seed motif. Nat Commun 4: 2730 2013.

13. Chevalier B, Adamiok A, Mercey O, Revinski DR, Zaragosi LE, Pasini A, Kodjabachian L, Barbry P and Marcet B: miR-34/449 control apical actin network formation during multiciliogenesis through small GTPase pathways. Nat Commun 6: 8386, 2015.

14. Han YC, Vidigal JA, Mu P, Yao E, Singh I, González AJ, Concepcion CP, Bonetti C, Ogrodowski P, Carver B, et al: An allelic series of miR-17 92-mutant mice uncovers functional specialization and cooperation among members of a microRNA polycistron. Nat Genet 47: 766-775, 2015.
15. Marzi MJ, Puggioni EM, Dall'Olio V, Bucci G, Bernard L, Bianchi F, Crescenzi M, Di Fiore PP and Nicassio F: Differentiation-associated microRNAs antagonize the Rb-E2F pathway to restrict proliferation. J Cell Biol 199: 77-95, 2012.

16. Rokavec M, Öner MG, Li H, Jackstadt R, Jiang L, Lodygin D, Kaller M, Horst D, Ziegler PK, Schwitalla S, et al: IL-6R/STAT3/miR-34a feedback loop promotes EMT-mediated colorectal cancer invasion and metastasis. J Clin Invest 124 $1853-1867,2014$

17. Rokavec M, Öner MG, Li H, Jackstadt R, Jiang L, Lodygin D, Kaller M, Horst D, Ziegler PK, Schwitalla S, et al: Corrigendum. IL-6R/STAT3/miR-34a feedback loop promotes EMT-mediated colorectal cancer invasion and metastasis. J Clin Invest 125: $1362,2015$.

18. Calin GA and Croce CM: MicroRNA signatures in human cancers. Nat Rev Cancer 6: 857-866, 2006.

19. Lu J, Getz G, Miska EA, Alvarez-Saavedra E, Lamb J, Peck D, Sweet-Cordero A, Ebert BL, Mak RH, Ferrando AA, et al: MicroRNA expression profiles classify human cancers. Nature 435: 834-838, 2005.

20. Kulshreshtha R, Ferracin M, Wojcik SE, Garzon R, Alder H, Agosto-Perez FJ, Davuluri R, Liu CG, Croce CM, Negrini M, et al: A microRNA signature of hypoxia. Mol Cell Biol 27: 1859-1867, 2007.

21. Agrawal R, Pandey P, Jha P, Dwivedi V, Sarkar C and Kulshreshtha R: Hypoxic signature of microRNAs in glioblastoma: Insights from small RNA deep sequencing. BMC Genomics 15: 686, 2014.

22. Xu X, Jia R, Zhou Y, Song X, Wang J, Qian G, Ge S and Fan X: Microarray-based analysis: Identification of hypoxia-regulated microRNAs in retinoblastoma cells. Int J Oncol 38: 1385-1393, 2011.

23. Tsai WC, Hsu SD, Hsu CS, Lai TC, Chen SJ, Shen R, Huang Y, Chen HC, Lee CH, Tsai TF, et al: MicroRNA-122 plays a critical role in liver homeostasis and hepatocarcinogenesis. J Clin Invest 122: 2884-2897, 2012.

24. Hsu SH, Wang B, Kota J, Yu J, Costinean S, Kutay H, Yu L, Bai S, La Perle K, Chivukula RR, et al: Essential metabolic, anti-inflammatory, and anti-tumorigenic functions of miR-122 in liver. J Clin Invest 122: 2871-2883, 2012. 